\title{
Pattern avoidance in the rook monoid
}

\author{
Daniel Daly and Lara PudWell
}

\begin{abstract}
We consider two types of pattern avoidance in the rook monoid, i.e. the set of $0-1$ square matrices with at most one nonzero entry in each row and each column. For one-dimensional rook patterns, we completely characterize monoid elements avoiding a single pattern of length at most three and develop an enumeration scheme algorithm to study rook placements avoiding sets of patterns.
\end{abstract}

KEYWORDS AND PHRASES: Pattern avoidance, rook monoid, enumeration schemes.

\section{Introduction}

The rook monoid $\mathcal{R}_{n}$ is the monoid consisting of $0-1$ square matrices such that every row and column contains at most one nonzero entry. Such an $n \times n$ matrix may be viewed as an encoding for an arrangement of non-attacking rooks on an $n \times n$ board where a 1 denotes a rook and a 0 denotes an empty square. An example $0-1$ matrix and its corresponding rook placement are shown in Figure 1.

Combinatorially, rook monoid elements can be viewed as repetition-free words in the poset $\{1,2,3, \ldots\}+\{0\}$ where 0 is incomparable to any nonzero number. In particular, $\pi \in \mathcal{R}_{n}$ can be represented as a word $\pi=\pi_{1} \cdots \pi_{n}$ with the properties that for all $1 \leq i \leq n, \pi_{i} \in\{0,1, \ldots, n\}$ and if $\pi_{i}=\pi_{j}>$ 0 then $i=j$. Here, $\pi_{i}=0$ indicates column $i$ is empty and $\pi_{i}=j>0$ indicates a rook in column $i$, row $j$. For example, $\mathcal{R}_{2}=\{00,01,02,10,20,12,21\}$, corresponding to the 7 rook placements in Figure 2. Similarly, the rook placement in Figure 1 has word encoding 5470203. Superficially, a rook placement may look like a partial permutation in the sense of Claesson, Jelínek, Jelínková, and Kitaev [5], but the zeros in rook placements have a different interpretation than the holes in partial permutations.

Rook monoids have also been the focus of recent algebraic interest as generalizations of symmetric groups. Can and Renner gave a combinatorial description of the Bruhat-Chevalley order on rook monoids in [4]. In addition, Halverson and Ram studied the representation theory of the $q$-rook 


$\left[\begin{array}{lllllll}0 & 0 & 1 & 0 & 0 & 0 & 0 \\ 0 & 0 & 0 & 0 & 0 & 0 & 0 \\ 1 & 0 & 0 & 0 & 0 & 0 & 0 \\ 0 & 1 & 0 & 0 & 0 & 0 & 0 \\ 0 & 0 & 0 & 0 & 0 & 0 & 1 \\ 0 & 0 & 0 & 0 & 1 & 0 & 0 \\ 0 & 0 & 0 & 0 & 0 & 0 & 0\end{array}\right]$

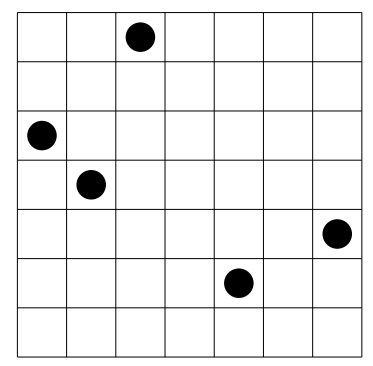

Figure 1: A 0-1 square matrix and its corresponding rook placement.

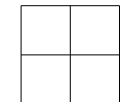

$\pi=00$

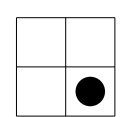

$\pi=01$

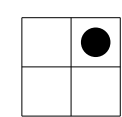

$\pi=02$

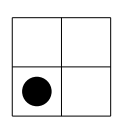

$\pi=10$

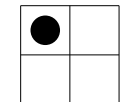

$\pi=20$

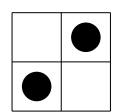

$\pi=12 \quad \pi=21$

Figure 2: The rook placements and word encodings in $\mathcal{R}_{2}$.

monoid algebra in [8]. This paper will define and study two notions of pattern avoidance on rook monoids. In studying avoidance on rook monoids, it is possible that such notions will prove useful in studying the BruhatChevalley order on rook monoids in the same spirit as Tenner's results for the Bruhat order on the symmetric group [17]. However, this paper focuses on enumerative results rather than algebraic ones.

The study begins with the total number of rook monoid elements.

Theorem 1. For $n \geq 0,\left|\mathcal{R}_{n}\right|=\sum_{k=0}^{n}\left(\begin{array}{l}n \\ k\end{array}\right)^{2} k !$.

Proof. Let $k$ be the number of rooks in an element of $\mathcal{R}_{n}$. Clearly $0 \leq k \leq n$. Choose the positions and values of the nonzero entries in $\left(\begin{array}{l}n \\ k\end{array}\right)^{2}$ ways, and order these entries in $k$ ! ways.

The exponential generating function $\sum_{n=0}^{\infty}\left|\mathcal{R}_{n}\right| \frac{x^{n}}{n !}$ has a particularly attractive form, as standard manipulation reveals:

$$
\sum_{n=0}^{\infty}\left|\mathcal{R}_{n}\right| \frac{x^{n}}{n !}=\sum_{n=0}^{\infty} \sum_{k=0}^{n}\left(\begin{array}{l}
n \\
k
\end{array}\right)^{2} k ! \frac{x^{n}}{n !}=\sum_{n=0}^{\infty} \sum_{k=0}^{n}\left(\begin{array}{l}
n \\
k
\end{array}\right)^{2}(n-k) ! \frac{x^{n}}{n !}
$$

After changing the order of summation, simplifying factorials, and making 
the substitution $i=n-k$

$$
=\sum_{k=0}^{\infty} \frac{x^{k}}{k !} \sum_{n=k}^{\infty}\left(\begin{array}{l}
n \\
k
\end{array}\right) x^{n-k}=\sum_{k=0}^{\infty} \frac{x^{k}}{k !} \sum_{n=k}^{\infty}\left(\begin{array}{c}
i+k \\
k
\end{array}\right) x^{i} .
$$

Now, further simplify to obtain

$$
=\sum_{k=0}^{\infty} \frac{x^{k}}{k !} \frac{1}{(1-x)^{k+1}}=\sum_{k=0}^{\infty} \frac{\left(\frac{x}{1-x}\right)^{k}}{(1-x) k !}=\frac{e^{\left(\frac{x}{1-x}\right)}}{1-x} .
$$

Let $r_{n, k}=\left(\begin{array}{l}n \\ k\end{array}\right)^{2} k$ ! be the number of rook placements on an $n \times n$ board with exactly $k$ rooks. The polynomials $R_{n}(x)=\sum_{k=0}^{n} r_{n, k} x^{k}$ are well-studied (see, for example, $[7,9,15])$. They are:

$$
\begin{aligned}
& R_{1}(x)=x+1 \\
& R_{2}(x)=2 x^{2}+4 x+1 \\
& R_{3}(x)=6 x^{3}+18 x^{2}+9 x+1, \\
& R_{4}(x)=24 x^{4}+96 x^{3}+72 x^{2}+16 x+1, \\
& R_{n}(x)=n ! x^{n} L_{n}^{(m-n)}\left(-x^{-1}\right) .
\end{aligned}
$$

where $L_{n}^{\sigma}(x)$ is the generalized Laguerre polynomial. In this context, $\left|\mathcal{R}_{n}\right|=$ $R_{n}(1)$.

Now that $\left|\mathcal{R}_{n}\right|$ has been computed, it is time to introduce rook patterns and the corresponding notation.

First, consider the notion of pattern avoidance in permutations. The set of permutations of length $n$, denoted $\mathcal{S}_{n}$, is a proper subset of $\mathcal{R}_{n}$. That is, $\mathcal{S}_{n}$ is the set of $n \times n$ 0-1 matrices with exactly one 1 in each row and each column. Here, $\pi \in \mathcal{S}_{n}$ contains $\rho \in \mathcal{S}_{m}$ if and only if there exist $1 \leq i_{1}<$ $i_{2}<\cdots<i_{m} \leq n$ such that $\pi_{i_{a}}>\pi_{i_{b}}$ if and only if $\rho_{a}>\rho_{b}$. In this case, $\pi_{i_{1}} \cdots \pi_{i_{m}}$ is said to be order-isomorphic to $\rho$. Let

$$
\mathcal{S}_{n}(Q)=\left\{\pi \in \mathcal{S}_{n} \mid \pi \text { avoids } \rho \text { for all } \rho \in Q\right\},
$$

and $\mathrm{s}_{n}(Q)=\left|\mathcal{S}_{n}(Q)\right|$. The quantities $\mathrm{s}_{n}(Q)$ are well-known for a variety of choices of $Q$.

Now, since $\mathcal{R}_{n}$ is a superset of $\mathcal{S}_{n}$, the classical definition of permutation pattern generalizes to incorporate the zeros of rook monoid elements. 
Definition 1. Let $\pi \in \mathcal{R}_{n}$ and $\rho \in \mathcal{R}_{m} . \pi$ contains $\rho$ as a (one-dimensional) pattern if there exist $1 \leq i_{1}<i_{2}<\cdots<i_{m} \leq n$ such that $\pi_{i_{\ell}}=0$ if and only if $\rho_{\ell}=0$ and for $\pi_{i_{a}}, \pi_{i_{b}}>0, \pi_{i_{a}}>\pi_{i_{b}}$ if and only if $\rho_{a}>$ $\rho_{b}$. Equivalently, $\pi_{i_{1}} \cdots \pi_{i_{m}}$ and $\rho$ have corresponding zeros and the nonzero elements of $\pi_{i_{1}} \cdots \pi_{i_{m}}$ are order-isomorphic to the nonzero elements of $\rho$. If $\pi$ does not contain $\rho$, then $\pi$ avoids $\rho$.

For example, 0104023 contains 0312 as evidenced by 0423. Similarly, 00102 contains 102 and 12 but not 1002. 00102 also avoids 123; although 012 is an increasing subword of length 3, zeros are treated differently than nonzero elements.

Now, let

$$
\mathcal{R}_{n, k}(Q)=\left\{\pi \in \mathcal{R}_{n} \mid \pi \text { avoids } \rho \text { for all } \rho \in Q, \pi \text { has exactly } k \text { rooks }\right\}
$$

$\mathrm{r}_{n, k}(Q)=\left|\mathcal{R}_{n, k}(Q)\right|, \mathcal{R}_{n}(Q)=\bigcup_{0<k<n} \mathcal{R}_{n, k}(Q)$ and $\mathrm{r}_{n}(Q)=\left|\mathcal{R}_{n}(Q)\right|$. For fixed $n$ and $Q, \mathcal{S}_{n}=\mathcal{R}_{n, n} \subset \mathcal{R}_{n}$ and $\overline{\mathcal{S}}_{n}(Q)=\mathcal{R}_{n, n}(Q) \subseteq \mathcal{R}_{n}(Q)$. Therefore, a lower bound for $\mathrm{r}_{n}(Q)$ exists since $\mathrm{s}_{n}(Q) \leq \mathrm{r}_{n}(Q)$.

The primary goal of this paper is to determine $\mathrm{r}_{n}(Q)$ and $\mathrm{r}_{n, k}(Q)$ for a variety of pattern sets $Q$ and explore their connections with other combinatorial objects. First, note that some rook placements are already equivalent as patterns in the one-dimensional sense. For example, 20 and 10 are equivalent rook patterns since their nonzero subwords are order-isomorphic. Similarly, the elements 045012 and 035012 are both equivalent to 034012 . For simplicity, only reduced rook patterns, i.e. those where all empty rows are at the top of the board, will be considered. For example, the reduced rook patterns in $\mathcal{R}_{2}$ are $00,10,01,12$, and 21.

Next, since rook placements are drawn on square boards, using the symmetries of the square will further simplify the computation. Let $\pi^{r}=$ $\pi_{n} \cdots \pi_{1}$ denote the reversal of $\pi$. Let $c_{n}\left(\pi_{i}\right)=\left\{\begin{array}{ll}n+1-\pi_{i} & \pi_{i}>0 \\ 0 & \pi_{i}=0\end{array}\right.$ and let $\pi^{c}=c_{n}\left(\pi_{1}\right) c_{n}\left(\pi_{2}\right) \cdots c_{n}\left(\pi_{n}\right)$ denote the complement of $\pi$. Then. $\mathrm{r}_{n, k}(Q)=$ $\mathrm{r}_{n, k}\left(\left\{q^{r} \mid q \in Q\right\}\right)=\mathrm{r}_{n, k}\left(\left\{q^{c} \mid q \in Q\right\}\right)=\mathrm{r}_{n, k}\left(\left\{q^{r c} \mid q \in Q\right\}\right)$. The symmetry of the inverse is lost since inverses map empty columns to empty rows, and the one-dimensional definition of avoidance respects empty columns but not empty rows. Two pattern sets $Q_{1}$ and $Q_{2}$ where $\mathrm{r}_{n, k}\left(Q_{1}\right)=\mathrm{r}_{n, k}\left(Q_{2}\right)$ for $n, k \geq 0$ are said to be Wilf equivalent. Two pattern sets that are Wilf equivalent via the reverse and/or complement maps are said to be trivially Wilf equivalent. Making use of these equivalences, there are three trivial Wilf classes for reduced rook patterns in $\mathcal{R}_{2}$. A representative of each class is 00 , 01 , and 12 . 
Section 2 completely classifies rook placements avoiding a single pattern of length at most three. Section 3 considers an algorithmic approach to compute $\mathrm{r}_{n}(Q)$ and $\mathrm{r}_{n, k}(Q)$. Finally, Section 4 introduces an even more general type of rook pattern analogous to bivincular permutation patterns.

\section{Basic enumeration}

In this section, rook placements avoiding a single pattern of length 1,2 , or 3 are completely classified. Brute force computation reveals that the number of trivial Wilf equivalence classes of length one patterns is two, of length two patterns is three, and of length three patterns is seven.

The basic enumeration begins with three results that apply to patterns of any length $m$, but of special types: patterns with $m$ zeros, patterns with $m-1$ zeros, and patterns with no zeros. After studying these three pattern types, the remaining patterns of length at most three will be handled individually.

Throughout this section, the notation $0^{m}$ indicates a string of $m$ consecutive 0s.

\subsection{Special pattern types}

\subsubsection{The case of $m$ zeros}

Theorem 2. Let $0 \leq k \leq n$ and $m \geq 0$. Then,

$$
\mathrm{r}_{n, k}\left(0^{m}\right)=\left\{\begin{array}{ll}
\left(\begin{array}{l}
n \\
k
\end{array}\right)^{2} k ! & k \geq n-m+1 \\
0 & k \leq n-m
\end{array},\right.
$$

and thus, $\mathrm{r}_{n}\left(0^{m}\right)=\sum_{k=n-m+1}^{n}\left(\begin{array}{l}n \\ k\end{array}\right)^{2} k !$.

Proof. This proof is the same as the proof of Theorem 1 except there are at most $m-1$ zeros.

The sum $\mathrm{r}_{n}\left(0^{m}\right)=\sum_{k=n-m+1}^{n}\left(\begin{array}{l}n \\ k\end{array}\right)^{2} k !$ simplifies nicely for small values of $m$. In particular:

$$
\begin{gathered}
\mathrm{r}_{n}(0)=n ! \\
\mathrm{r}_{n}(00)=(n+1) ! \\
\mathrm{r}_{n}(000)=\frac{1}{4} n !\left(n^{2}+3 n+4\right),
\end{gathered}
$$




$$
\mathrm{r}_{n}(0000)=\frac{1}{36} n !\left(n^{3}+6 n^{2}+29 n+36\right) .
$$

The first two of these results make immediate sense: only the permutations of length $n$ avoid 0 , and permutations of a subset of size $n$ chosen from $\{0,1, \ldots, n\}$ avoid 00 . As $m$ increases, the resulting enumeration formulas are always of the form

$$
\mathrm{r}_{n}\left(0^{m}\right)=\frac{1}{((m-1) !)^{2}} n !\left(P_{m}(n)\right)
$$

where $P_{m}(n)$ is a polynomial in $n$ of degree $m-1$.

This enumeration can be expressed more simply using exponential generating functions. To that end, let $f_{m}(x)=\sum_{n=0}^{\infty} \mathrm{r}_{n}\left(0^{m}\right) \frac{x^{n}}{n !}$. Since $\mathrm{r}_{n}(0)=n$ !, $f_{1}(x)=\sum_{n=0}^{\infty} \mathrm{r}_{n}(0) \frac{x^{n}}{n !}=\sum_{n=0}^{\infty} x^{n}=\frac{1}{1-x}$. Now, the difference $\mathrm{r}_{n}\left(0^{m+1}\right)-$ $\mathrm{r}_{n}\left(0^{m}\right)$ counts elements of $\mathcal{R}_{n}$ with exactly $m$ zeros. From earlier computation that there are $\left(\begin{array}{l}n \\ m\end{array}\right)^{2}(n-m)$ ! such elements. Therefore

$$
f_{2}(x)-f_{1}(x)=\sum_{n=0}^{\infty}\left(\begin{array}{l}
n \\
1
\end{array}\right)^{2}(n-1) ! \frac{x^{n}}{n !}=\sum_{n=0}^{\infty} n x^{n}=\frac{x}{(1-x)^{2}} .
$$

Similarly

$$
\begin{aligned}
f_{3}(x)-f_{2}(x) & =\sum_{n=0}^{\infty}\left(\begin{array}{l}
n \\
2
\end{array}\right)^{2}(n-2) ! \frac{x^{n}}{n !} \\
& =\sum_{n=0}^{\infty}\left(\frac{n(n-1)}{2}\right)^{2} \frac{x^{n}}{n(n-1)} \\
& =\sum_{n=0}^{\infty} \frac{1}{2 !^{2}} n(n-1) x^{n} \\
& =\frac{x^{2}}{2(1-x)^{3}} .
\end{aligned}
$$

And in general:

$$
\begin{aligned}
f_{m+1}-f_{m}(x) & =\sum_{n=0}^{\infty}\left(\begin{array}{c}
n \\
m
\end{array}\right)^{2}(n-m) ! \frac{x^{n}}{n !} \\
& =\sum_{n=0}^{\infty}\left(\frac{n \cdots(n-(m-1))}{m !}\right)^{2} \frac{x^{n}}{n \cdots(n-(m-1))}
\end{aligned}
$$




$$
=\sum_{n=0}^{\infty} \frac{1}{m !^{2}} n(n-1) \cdots(n-(m-1)) x^{n}=\frac{x^{m}}{m !(1-x)^{m+1}} .
$$

Therefore,

$$
f_{m}(x)=\sum_{i=1}^{m} \frac{x^{i-1}}{(i-1) !(1-x)^{i}}=\frac{\Gamma\left(m, \frac{x}{1-x}\right) e^{\left(\frac{x}{1-x}\right)}}{(1-x)(m-1) !}
$$

where $\Gamma(m, z):=\int_{z}^{\infty} e^{-t} t^{m-1} d t$ is the incomplete Gamma function and the last equality comes from Maple simplification. In general, the $e^{\left(\frac{x}{1-x}\right)}$ in the numerator cancels with a $e^{-\left(\frac{x}{1-x}\right)}$ factor in $\Gamma\left(m, \frac{x}{1-x}\right)$ to produce a rational function in $x$.

2.1.2. The case of $\boldsymbol{m}-\mathbf{1}$ zeros and one $\mathbf{1}$ There are $m$ rook patterns of length $m$ with exactly one nonzero digit. For any fixed $m$, all such patterns are Wilf equivalent.

Theorem 3. Let $0 \leq j<\ell<m \leq n$. Then $\mathrm{r}_{n}\left(0^{j} 10^{m-j-1}\right)=\mathrm{r}_{n}\left(0^{\ell} 10^{m-\ell-1}\right)$. Further, for $0 \leq k \leq n, \mathrm{r}_{n, k}\left(0^{j} 10^{m-j-1}\right)=\mathrm{r}_{n, k}\left(0^{\ell} 10^{m-\ell-1}\right)$.

Proof. The goal is to provide a bijection $\xi_{j}$ from the set of elements of $\mathcal{R}_{n}$ that contain $0^{j} 10^{m-j-1}$ to the set of elements of $\mathcal{R}_{n}$ that contain $0^{j+1} 10^{m-j-2}$, which shows $\mathrm{r}_{n}\left(0^{j} 10^{m-j-1}\right)=\mathrm{r}_{n}\left(0^{\ell} 10^{m-\ell-1}\right)$ when $\ell=j+1$. Further, since such a bijection preserves the number of rooks in $\pi$,

$$
\mathrm{r}_{n, k}\left(0^{j} 10^{m-j-1}\right)=\mathrm{r}_{n, k}\left(0^{j+1} 10^{m-(j+1)-1}\right) .
$$

Then, by transitivity, the theorem holds for any choice of $j$ and $\ell$.

First, if $\pi \in \mathcal{R}_{n}$ contains both patterns, then define $\xi_{j}(\pi)=\pi$.

For $j \geq 1$, assume $\pi$ contains $0^{j} 10^{m-j-1}$ but avoids $0^{j+1} 10^{m-j-2}$. Consider the location of the $j^{\text {th }}$ zero and the $j+1^{\text {st }}$ zero in $\pi$. Write $\pi=x 0 y 0 z$ where $x$ and $z$ are words on $\{0, \ldots, n\}, y$ is a word on $\{1, \ldots, n\}$, the 0 between $x$ and $y$ is the $j^{\text {th }}$ zero of $\pi$ and the 0 between $y$ and $z$ is the $j+1^{\text {st }}$ zero of $\pi$. In order for $\pi$ to contain $0^{j} 10^{m-j-1}, z$ must have at least $m-j-2$ zeros. However, $z$ must also avoid $10^{m-j-2}$ in order for $\pi$ to avoid $0^{j+1} 10^{m-j-2}$. Hence, any copy of $0^{j} 10^{m-j-1}$ must contain a digit from $y$ and $z$. Now, $z$ contains at least $m-j-2$ zeros, but may contain more. Keep the rightmost $m-j-2$ zeros fixed in $z$ and then move all extra zeros along with the $j+1^{\text {st }}$ zero directly to the left of $y$. Define this new element to be $\xi_{j}(\pi)$. 
To invert this map, suppose that $\pi$ contains $0^{j+1} 10^{m-j-2}$ and avoids $0^{j} 10^{m-j-1}$. Then $\pi^{r}$ contains $0^{m-j-2} 10^{j+1}$ and avoids $0^{m-j-1} 10^{j}$. Further, $\xi_{m-j-2}(\pi)$ contains $0^{m-j-1} 10^{j}$ and avoids $0^{m-j-2} 10^{j+1}$, so $\xi_{m-j-2}(\pi)^{r}$ contains $0^{j} 10^{m-j-1}$ and avoids $0^{j+1} 10^{m-j-2}$.

For $j=0,10^{m-1}$ is equivalent to $0^{m-1} 1$ by reversal, and $0^{m-1} 1$ is equivalent to all other patterns of the form $0^{j} 10^{m-j-1}$ by the argument above.

As an example of the procedure outlined above, consider the rook monoid element 0012300040 which contains 001000 and avoids 000100 . The procedure decomposes the rook monoid element as 0(0)123(0)0040 and then swaps the 123 with the two zeros directly to the right to obtain 0000123040.

Since $\mathrm{r}_{n}(\rho)=\mathrm{r}_{n}\left(0^{m-1} 1\right)$ for all $\rho \in \mathcal{R}_{m}$ with $m-1$ zeros and one 1 , it suffices to compute $\mathrm{r}_{n}\left(0^{m-1} 1\right)$ exactly.

Theorem 4. For $n \geq 0, \mathrm{r}_{n}(01)=\mathrm{r}_{n}(10)=\sum_{j=0}^{n} \frac{n !}{j !}$.

Proof. A 01-avoiding rook monoid element consists of some permutation of $i$ nonzero entries followed by $n-i$ zeros for $0 \leq i \leq n$. Choose $i$ nonzero elements in $\left(\begin{array}{c}n \\ i\end{array}\right)$ ways, permute them in $i$ ! ways, and make the substitution $j=n-i$ to obtain the given formula.

An alternate explanation shows that $\mathrm{r}_{n}(01)=1+n \mathrm{r}_{n-1}(01)$ where the 1 counts the all zeros element, and the second term counts all other 01avoiders: there are $n$ nonzero choices for what the first nonzero digit may be, and $\mathrm{r}_{n-1}(01)$ 01-avoiding ways to arrange the remaining elements.

Standard algebraic manipulation reveals the exponential generating function $\sum_{n=0}^{\infty} \mathrm{r}_{n}(01) \frac{x^{n}}{n !}=\frac{e^{x}}{1-x}$. This is also sequence A000522 in the Online Encyclopedia of Integer Sequences (OEIS) [11] which has at least 21 different combinatorial interpretations.

Towards generality, let $\mathrm{r}_{n}^{p}(\rho)$ be the number of elements of $\mathcal{R}_{n}(\rho)$ beginning with pattern $p$ and suppose $\rho=0^{j} 1$ where $j>1$. For $n \geq i$,

$$
\begin{aligned}
& \mathrm{r}_{n}\left(0^{j} 1\right)=\mathrm{r}_{n}^{0}\left(0^{j} 1\right)+n \mathrm{r}_{n-1}\left(0^{j} 1\right), \\
& \mathrm{r}_{n}^{0^{i}}\left(0^{j} 1\right)=\mathrm{r}_{n}^{0^{i+1}}\left(0^{j} 1\right)+n \mathrm{r}_{n-1}^{0^{i}}\left(0^{j} 1\right) \text { for } 1 \leq i \leq j-2, \\
& \mathrm{r}_{n}^{0^{i}}\left(0^{j} 1\right)=1+n \mathrm{r}_{n-1}^{0^{i}}\left(0^{j} 1\right) \text { for } i=j-1 .
\end{aligned}
$$

For fixed $j$,

$$
\sum_{n \geq 0} \mathrm{r}_{n}^{0^{j-1}}\left(0^{j} 1\right) \frac{x^{n}}{n !}=\frac{e^{x}-\sum_{i=0}^{j-2} x^{i} / i !}{1-x}
$$


which can be used to recursively derive the exponential generating function for each sequence $\mathrm{r}_{n}^{0^{i}}\left(0^{j} 1\right)$ for $i=j-2, j-3, \ldots 0$.

Completing this process for $j=2, j=3$, and $j=4$, gives, for example,

$$
\begin{gathered}
\sum_{n \geq 0} \mathrm{r}_{n}(001) \frac{x^{n}}{n !}=\frac{e^{x}-x}{(1-x)^{2}}(\text { OEIS A193657), } \\
\sum_{n \geq 0} \mathrm{r}_{n}(0001) \frac{x^{n}}{n !}=\frac{e^{x}-2 x}{(1-x)^{3}}, \\
\sum_{n \geq 0} \mathrm{r}_{n}(00001) \frac{x^{n}}{n !}=\frac{e^{x}-3 x+x^{2}-x^{3} / 2}{(1-x)^{4}}
\end{gathered}
$$

Next, consider rook placements that avoid a pattern of the form $0^{j} 1$ and contain exactly $k$ rooks.

Theorem 5. For $0 \leq k \leq n, \mathrm{r}_{n, k}(01)=\left(\begin{array}{l}n \\ k\end{array}\right) k !=\frac{n !}{(n-k) !}$.

Proof. As in the proof of Theorem 4, all $n-k$ empty columns must be at the end of the rook element in order to avoid 01. Thus, choose which $k$ rows to put rooks in in one of $\left(\begin{array}{l}n \\ k\end{array}\right)$ ways and then place a permutation of length $k$ in the intersection of these $k$ rows and the first $k$ columns.

Theorem 6. For $0 \leq k \leq n$,

$$
\mathrm{r}_{n, k}(001)=\left\{\begin{array}{ll}
\left(\begin{array}{l}
n \\
k
\end{array}\right)^{2} k ! & k \geq n-1 \\
\left(\begin{array}{l}
n \\
k
\end{array}\right)(k+1) ! & k \leq n-2
\end{array} .\right.
$$

Proof. To avoid 001 there are two cases. If there are no empty columns or there is exactly one empty column (i.e. $k=n$ or $k=n-1$ ), it is impossible to have a 001 pattern, so choose which $k$ rows and which $k$ columns have rooks in one of $\left(\begin{array}{l}n \\ k\end{array}\right)^{2}$ ways, and then fill a permutation of length $k$ into the intersection of these rows and columns.

If there are two or more columns (i.e. $k \leq n-2$ ), then all but one empty column is at the end of the rook element. In this case, choose the $k$ rows to put rooks in in one of $\left(\begin{array}{l}n \\ k\end{array}\right)$ ways, choose which one of the first $k+1$ columns is empty in one of $(k+1)$ ways, and fill a permutation of length $k$ into the selected non-empty rows and columns in one of $k$ ! ways. 
2.1.3. The case of permutation patterns When avoiding a pattern with no zeros, the following theorem holds.

Theorem 7. Consider $\rho \in \mathcal{S}_{m}$. Then $\mathrm{r}_{n}(\rho)=\sum_{k=0}^{n}\left(\begin{array}{l}n \\ k\end{array}\right)^{2} \mathrm{~s}_{k}(\rho)$.

Proof. This proof is the same as the proof of Theorem 1 except there are only $\mathrm{s}_{k}(\rho)$ ways to arrange the nonzero digits such that they still avoid $\rho$.

As a consequence of Theorem 7 ,

$$
\begin{gathered}
\mathrm{r}_{n}(12)=\mathrm{r}_{n}(21)=\sum_{k=0}^{n}\left(\begin{array}{l}
n \\
k
\end{array}\right)^{2}=\left(\begin{array}{c}
2 n \\
n
\end{array}\right), \text { and } \\
\mathrm{r}_{n}(123)=\mathrm{r}_{n}(132)=\mathrm{r}_{n}(213)=\mathrm{r}_{n}(231)=\mathrm{r}_{n}(312)=\mathrm{r}_{n}(321)=\sum_{k=0}^{n}\left(\begin{array}{l}
n \\
k
\end{array}\right)^{2} C_{k},
\end{gathered}
$$

where $C_{k}$ is the $k^{\text {th }}$ Catalan number. The latter sequence is OEIS sequence A086618.

In general, the Wilf classes for permutation pattern avoidance in the rook monoid correspond precisely to the Wilf classes for permutation pattern avoidance in the set of permutations.

\subsection{Wilf-classification of patterns of length at most three}

The results of the previous subsection can now be used to complete the Wilf classification of rook patterns of length at most three. There are precisely two patterns of length one (0 and 1$)$, and five patterns of length two which fall into three trivial equivalence classes $(\{00\},\{01,10\}$, and $\{12,21\})$. All of these patterns are addressed by Theorems 2, 4, and 7. There are 16 patterns of length three, and they fall into seven trivial equivalence classes. A representative of each of the seven classes is 000, 001, 010, 123, 132, 012, and 102. Again, our previous work accounts for five of the seven classes. It remains to determine $\mathrm{r}_{n, k}(012)$ and $\mathrm{r}_{n, k}(102)$.

Theorem 8. For $0 \leq k \leq n$,

$$
\mathrm{r}_{n, k}(012)=\left\{\begin{array}{ll}
n ! & k=n \\
\sum_{j=1}^{k+1}\left(\begin{array}{c}
n-j \\
n-k-1
\end{array}\right)\left(\begin{array}{l}
n \\
k
\end{array}\right)\left(\begin{array}{c}
k \\
j-1
\end{array}\right)(j-1) ! & k \leq n-1
\end{array} .\right.
$$


Proof. For avoiding 012, there are two cases. If there are no empty columns (i.e. $k=n$ ), it is impossible to contain a 012 pattern, and there are $n$ ! permutations.

If $k<n$, then avoiding 012 means that after the first empty column, all elements appear in decreasing order. In this case, let $j$ be the location of the first empty column $(1 \leq j \leq k+1)$. Choose the locations of the remaining $n-k-1$ empty columns in $\left(\begin{array}{c}n-j \\ n-k-1\end{array}\right)$ ways. Choose the non-empty rows in $\left(\begin{array}{l}n \\ k\end{array}\right)$ ways. Choose which of those $k$ non-empty rows has a rook before column $j$ in $\left(\begin{array}{c}k \\ j-1\end{array}\right)$ ways, and put a permutation of length $(j-1)$ ! in the non-empty rows and columns before column $j$. The locations of the remaining rooks are uniquely determined by the rows and columns that were selected to be non-empty but do not yet have a rook; those rooks must appear in these rows and columns in decreasing order.

Theorem 9. For $0 \leq k \leq n$,

$$
\mathrm{r}_{n, k}(102)=\left\{\begin{array}{ll}
n ! & k=n \\
\sum_{P}\left(\begin{array}{l}
n \\
k
\end{array}\right)(\Delta P) ! & k \leq n-1
\end{array},\right.
$$

where the sum is over sets $P=\left\{p_{1}, \ldots, p_{n-k}\right\} \subseteq\{1, \ldots, n\}$ where $1 \leq p_{1}<$ $p_{2}<\cdots<p_{n-k} \leq n$ and $(\Delta P) !:=\left(p_{1}-1\right) !\left(p_{2}-p_{1}-1\right) ! \cdots\left(p_{n-k}-p_{n-k-1}-\right.$ $1) !\left(n-p_{n-k}\right) !$.

Proof. For avoiding 102, there are two cases. If there are no empty columns (i.e. $k=n$ ), it is impossible to contain a 102 pattern, and there are $n$ ! permutations.

If $k<n$, then avoiding 102 means that all digits before a given empty column are larger than all digits after the column. Let $p_{1}, \ldots, p_{n-k}$ be the indices of the empty columns. Choose the $n-k$ empty columns, noting their locations, and then choose the $k$ non-empty rows in one of $\left(\begin{array}{l}n \\ k\end{array}\right)$ ways. Now, there are $p_{1}-1$ rooks before column $p_{1}$. These must be the largest $p_{1}-1$ digits, but they can appear in any order amongst themselves. Similarly, there are $p_{2}-p_{1}-1$ rooks between columns $p_{1}$ and $p_{2}$. These must be the next largest $p_{2}-p_{1}-1$ digits, but they can appear in any order amongst themselves. Similarly, the number of ways to place the rooks between consecutive empty columns $p_{i}$ and $p_{i+1}$ is $\left(p_{i+1}-p_{i}-1\right)$ ! for $1 \leq i \leq n-k-1$, so $(\Delta P)$ ! is the number of ways to arrange rooks in the selected columns.

Here, the $(\Delta P)$ ! notation for products of factorials of consective differences is used for compactness. It turns out that the triangle of values $\frac{r_{n, k}(102)}{\left(\begin{array}{l}n \\ k\end{array}\right)}$ 
Table 1: Sequences from avoiding a single pattern of length 1, 2, or 3

\begin{tabular}{|c|l|c|c|}
\hline Pattern $q$ & $r_{n}(q)$ & OEIS & Theorem \\
\hline \hline 1 & $1,1,1,1,1, \ldots$ & A000012 & Theorem 7 \\
\hline 0 & $1,2,6,24,120, \ldots$ & A000142 & Theorem 2 \\
\hline \hline 01 & $2,5,16,65,326, \ldots$ & A000522 & Theorem 4 \\
\hline 12 & $2,6,20,70,252, \ldots$ & A000984 & Theorem 7 \\
\hline 00 & $2,6,24,120,720, \ldots$ & A000142 & Theorem 2 \\
\hline \hline 102 & $2,7,31,159,916, \ldots$ & A221958 & Theorem 9 \\
\hline 012 & $2,7,31,159,921, \ldots$ & A221957 & Theorem 8 \\
\hline 001 (equivalently 010) & $2,7,31,165,1031, \ldots$ & A193657 & Theorem 6 \\
\hline 123 (equivalently 132) & $2,7,33,183,1118, \ldots$ & A086618 & Theorem 7 \\
\hline 000 & $2,7,33,192,1320, \ldots$ & A006595 & Theorem 2 \\
\hline
\end{tabular}

appears as A084938 in [11], where it has several other combinatorial interpretations.

Table 1 contains the enumeration data for $\mathrm{r}_{n}(Q)$ where $Q$ is a single pattern of length at most three. Notice that every single sequence in Table 1 is already in the OEIS. Of the nine distinct sequences cited within, two (OEIS A221957 and OEIS A221958) appear to be new to the literature. On the other hand, the seven previously-studied sequences offer ample opportunity to connect rook placements to other combinatorial objects. Two such objects (counted by OEIS A006595 and OEIS A086618) are studied in Section 2.3. Questions of asymptotic growth for these sequences appear in Section 2.4.

\subsection{Connections to other combinatorial objects}

2.3.1. A-reduced elements of $\boldsymbol{B}_{\boldsymbol{n}}$ From Section 2.1.1, $\sum_{n \geq 0} \mathrm{r}_{n}(000) \frac{x^{n}}{n !}=$ $\frac{x^{2}-2 x+2}{2(1-x)^{3}}$, which is the exponential generating function for $\frac{(n+\overline{2}) !}{4}+\frac{n !}{2}$. There is a connection between $\mathcal{R}_{n}(000)$ and a certain subset of $B_{n}$, the Coxeter group of type $B$, also known as the hyperoctahedral group, which consists of all signed permutations of $[n]$. First, a signed permutation is a permutation where each number may or may not have a bar over it. For example, there are eight such permutations of length two: $B_{2}=\{12, \overline{1} 2,1 \overline{2}, \overline{12}, 21, \overline{2} 1,2 \overline{1}, \overline{21}\}$. A copy of a pattern means that the underlying numbers are order-isomorphic and the bars match. The reader is referred to [3] for more information on Coxeter groups. Stembridge gives a simple combinatorial description of Areduced elements of $B_{n}$ in Theorem 2.3 of [16], i.e. an element of $B_{n}$ is $A$-reduced if and only if that element avoids the patterns $\overline{12}$ and $1 \overline{32}$.

Theorem 10. For all $n \geq 1$, the number of $A$-reduced elements of $B_{n}$ is equal to $\mathrm{r}_{n}(000)$. 
Proof. To prove this, we provide a bijection $\phi$ from $\mathcal{R}_{n}(000)$ to the set of all $A$-reduced elements of $B_{n}$. Let $\pi \in \mathcal{R}_{n}(000)$. Define $\phi(\pi)=\pi$ if $\pi$ contains no zeros.

If $\pi$ contains exactly one zero, then $\pi=\pi_{1} \cdots \pi_{i-1} 0 \pi_{i+1} \cdots \pi_{n}$ where $a \in[n]$ does not appear in $\pi$. Define $\phi(\pi)=\pi_{1} \cdots \pi_{i-1} \bar{a} \pi_{i+1} \cdots \pi_{n}$.

If $\pi$ contains exactly two zeros, then $\pi=\pi_{1} \cdots \pi_{i} 0 y 0 z$ where each member of $\pi_{1} \cdots \pi_{i}$ belongs to $[n]=\{1, \ldots, n\}$ and $y$ and $z$ are strings of elements of $[n]$. There are exactly two elements from $[n]$ that do not appear in $\pi$. Label them $a$ and $b$ and without loss of generality assume $a<b$. Let $I_{1}$ be the set $\{a, b\}$. If $\pi_{1}$ is less than $a$, then define $\pi_{1}^{\prime}=\bar{b}$ and $I_{2}=\left\{a, \pi_{1}\right\}$. If not, define $\pi_{1}^{\prime}=\pi_{1}$ and $I_{2}=I_{1}$. Now, if $\pi_{2}$ is less than the minimum element in $I_{2}$, define $\pi_{2}^{\prime}=\overline{\max \left(I_{2}\right)}$ and $I_{3}=\left\{\min \left(I_{2}\right), \pi_{2}\right\}$. Define $\pi_{2}^{\prime}=\pi_{2}$ and $I_{3}=I_{2}$ otherwise. Continue in this fashion for all $1<j \leq i$. For any $1<j \leq i$, define $\pi_{j}^{\prime}=\overline{\max \left(I_{j}\right)}$ and $I_{j+1}=\left\{\min \left(I_{j}\right), \pi_{j}\right\}$ if $\pi_{j}<\min \left(I_{j}\right)$ and $\pi_{j}^{\prime}=\pi_{j}$ and $I_{j+1}=I_{j}$ otherwise. Notice that for all $1 \leq j_{1}<j_{2} \leq i$, the elements of $I_{j_{1}}$ are always greater than or equal to the elements of $I_{j_{2}}$. Define $\phi(\pi)=\pi_{1}^{\prime} \pi_{2}^{\prime} \cdots \pi_{i}^{\prime} \overline{\max \left(I_{j}\right)} y \overline{\min \left(I_{j}\right)} z$.

By construction $\phi(\pi)$ avoids $\overline{12}$. Now, a $1 \overline{32}$ pattern can only occur in the subword $\pi_{1}^{\prime} \cdots \pi_{i}^{\prime} \overline{\max \left(I_{j}\right) \min \left(I_{j}\right)}$. The ' 1 ' in the $1 \overline{32}$ pattern must be $\pi_{j}^{\prime}$ for some $1 \leq j \leq i$ and hence there are two elements to the right of $\pi_{j}^{\prime}$ that are greater than $\pi_{j}^{\prime}$ and barred. Since $\pi_{j}^{\prime}$ is not barred, $\pi_{j}^{\prime}=\pi_{j}$ and, by the construction of the $I$ sets, $\pi_{j}$ is less than both of the elements in $I_{j}$ and hence $\pi_{j}^{\prime}$ would be barred which is a contradiction. Therefore $\phi(\pi)$ is an $A$-reduced element of $B_{n}$.

Define an inverse function $\psi$ from the $A$-reduced elements of $B_{n}$ to $\mathcal{R}_{n}$. Let $\pi$ be an $A$-reduced element of $B_{n}$. If $\pi$ contains no barred elements, define $\psi(\pi)$ to be $\pi$. Similarly, if $\pi$ contains exactly one barred element, then $\psi(\pi)$ returns $\pi$ with the barred element replaced with a 0 . Now write $\pi=\pi_{1} \cdots \pi_{i} y \pi_{j} z$ where $y$ and $z$ are (possibly empty) sequences of unbarred symbols in $[n]$ and $\pi_{i}$ and $\pi_{j}$ are the rightmost two occurrences of barred elements. As $\pi$ avoids $\overline{12}$ all of the barred elements occur in decreasing order. Define $\pi_{i}^{\prime}=0$ and $\pi_{j}^{\prime}=0$. Next define $\pi_{\ell}^{\prime}=\pi_{\ell}$ if $\pi_{\ell}$ is unbarred. Now, list all of the other barred elements of $\pi$ from left to right as $\pi_{\ell_{1}}, \pi_{\ell_{2}}, \ldots, \pi_{\ell_{m}}$. Define $\pi_{\ell_{b}}^{\prime}=\pi_{\ell_{b}+2}$ without the bar. Finally define $\psi(\pi)=\pi_{1}^{\prime} \pi_{2}^{\prime} \cdots \pi_{i}^{\prime} y \pi_{j}^{\prime} z$. As $\psi$ inverts $\phi, \phi$ is a bijection and the result follows.

As an example, consider $5312060 \in \mathcal{R}_{7}$. The construction of $\phi(5312060)$ is as follows: 


\begin{tabular}{c|c|c}
$n$ & element & $I_{n}$ \\
\hline 1 & 5312060 & $\{4,7\}$ \\
2 & 5312060 & $\{4,7\}$ \\
3 & $5 \overline{7} 12060$ & $\{3,4\}$ \\
4 & $5 \overline{74} 2060$ & $\{1,3\}$ \\
5 & $5 \overline{74} 2060$ & $\{1,3\}$ \\
6 & $5 \overline{74} 2 \overline{3} 6 \overline{1}$ & $\{1,3\}$
\end{tabular}

Hence $\phi(5312060)=5 \overline{74} 2 \overline{3} 6 \overline{1}$.

2.3.2. Reverse-complement invariant permutations Theorem 7 says that $\mathrm{r}_{n}(321)=\sum_{k=0}^{n}\left(\begin{array}{l}n \\ k\end{array}\right)^{2} C_{k}$ (OEIS A086618).

Let $\mathcal{S}_{2 n}^{r c}(q)$ be the number of permutations of length $2 n$ which are invariant under the reverse-complement map and avoid pattern $q$. Egge [6] showed that $\left|\mathcal{S}_{2 n}^{r c}(4321)\right|=\sum_{k=0}^{n}\left(\begin{array}{l}n \\ k\end{array}\right)^{2} C_{k}$. Egge's proof of this enumeration for reverse-complement-invariant permutations uses several bijections. Ultimately, he matches each member of $\mathcal{S}_{2 n}^{r c}(m(m-1) \cdots 21)$ to a 4-tuple $\left(P^{\prime}, Q^{\prime}, \pi_{o}, \pi_{e}\right)$. where $P^{\prime} \subseteq\{1, \ldots, n\}, Q^{\prime} \subseteq\{1, \ldots, n\},\left|P^{\prime}\right|=\left|Q^{\prime}\right|=k$ for some $0 \leq k \leq n, \pi_{o} \in \mathcal{S}_{k}\left(\left\lceil\frac{m+1}{2}\right\rceil \cdots 1\right)$, and $\pi_{e} \in \mathcal{S}_{n-k}\left(\left\lfloor\frac{m+1}{2}\right\rfloor \cdots 1\right)$. In the case where $m=4, \pi_{o} \in \mathcal{S}_{k}(321)$, and $\pi_{e} \in \mathcal{S}_{n-k}(21)$. In this event, notice that $\pi_{e}=12 \cdots(n-k)$, and so is uniquely determined once the value of $k$ is known. Making use of Egge's bijections yields the following result.

Theorem 11. $\mathcal{S}_{2 n}^{r c}(4321)$ is in bijection with $\mathcal{R}_{n}(321)$.

Proof. Let $P^{\prime}$ be the set of nonzero entries of an element of $R_{n}, Q^{\prime}$ be the positions of the nonzero entries of that element, and $\pi_{o}$ the permutation pattern formed by the nonzero entries of that element. This now extends Egge's bijection to elements of the rook monoid, providing a one-to-one correspondence with members of $\mathcal{S}_{2 n}^{r c}(4321)$.

\subsection{Asymptotic growth of rook sequences}

Finally, it is natural to consider the asymptotic growth of the sequences $\mathrm{r}_{n}(Q)$ in Table 1 . Recall that the exponential generating function for $r_{n}$ is $\frac{e^{\left(\frac{x}{1-x}\right)}}{1-x}$. Thus,

$$
r_{n}=n !\left[x^{n}\right] \frac{e^{\left(\frac{x}{1-x}\right)}}{1-x} \sim \frac{e^{2 \sqrt{n}-1 / 2}}{2 \sqrt{\pi} n^{1 / 4}} n !
$$


The $e^{2 \sqrt{n}}$ in the numerator exhibits much faster growth than the growth of pattern-avoiding rook sequences.

From Theorem 2,

$$
\mathrm{r}_{n}\left(0^{m}\right) \sim c n ! n^{m-1} .
$$

Similarly, using the exponential generating functions computed in Section 2.1.2, compute

$$
\begin{array}{r}
\mathrm{r}_{n}(01)=n !\left[x^{n}\right] \frac{e^{x}}{1-x} \sim e n !, \\
\mathrm{r}_{n}(001)=n !\left[x^{n}\right] \frac{e^{x}-x}{(1-x)^{2}} \sim e n n ! .
\end{array}
$$

Finally, from Theorem 7:

$$
\begin{aligned}
\mathrm{r}_{n}(1) & =1 \\
\mathrm{r}_{n}(12) & =\left(\begin{array}{c}
2 n \\
n
\end{array}\right) \sim \frac{4^{n}}{\sqrt{\pi n}} \\
\mathrm{r}_{n}(123) & =\sum_{j=0}^{n}\left(\begin{array}{l}
n \\
j
\end{array}\right)^{2} C_{j} \sim c \frac{9^{n}}{n^{2}} .
\end{aligned}
$$

Clearly, the number of rook placements avoiding $0^{m}$ or $0^{m-1} 1$ grows super-exponentially, and much more quickly than the number of rook placements avoiding a permutation pattern.

For patterns of length one, it is immediately clear that $\mathrm{r}_{n}(0)>>\mathrm{r}_{n}(1)$, and for patterns of length two, $\mathrm{r}_{n}(00)>>\mathrm{r}_{n}(01)>>\mathrm{r}_{n}(12)$, even though $\mathrm{r}_{n}(12)>\mathrm{r}_{n}(01)$ for small values of $n$.

For patterns of length three, $\mathrm{r}_{n}(000)>\mathrm{r}_{n}(001)>\mathrm{r}_{n}(123)$, again, even though $\mathrm{r}_{n}(123)>\mathrm{r}_{n}(001)$ for small values of $n$. The patterns 102 and 012 elicited sufficiently complicated formulas that precise asymptotics for these pattern classes remain open.

Nonetheless, these initial computations prompt one to conjecture that the all zeros pattern is the easiest pattern to avoid. This turns out to be the case for patterns of any length.

Theorem 12. Let $\rho \in \mathcal{R}_{m}$ be a rook pattern with exactly $i$ rooks where $i>0$. Then $\mathrm{r}_{n}\left(0^{m}\right)>\mathrm{r}_{n}(\rho)$ for sufficiently large $n$.

Proof. Let $\operatorname{co}_{n}(\rho)$ (resp. $\mathrm{co}_{n}\left(0^{m}\right)$ ) be the number of rook monoid elements of length $n$ that contain $\rho$ (resp. $\left.0^{m}\right)$. We will prove the equivalent statement 
that $\operatorname{co}_{n}\left(0^{m}\right)<\operatorname{co}_{n}(\rho)$ for sufficiently large $n$ by proving an even stronger statement.

Let $\mathrm{co}_{n, k}(\rho)$ (resp. $\mathrm{co}_{n, k}\left(0^{m}\right)$ be the number of rook monoid elements of $\mathcal{R}_{n, k}$ that contain $\rho$ (resp. $0^{m}$ ). We will prove that for $0 \leq k \leq n-$ $m, \mathrm{co}_{n, k}\left(0^{m}\right) \leq \operatorname{co}_{n, k+i}(\rho)$. Indeed, values outside of this range for $k$ are irrelevant. If $k>n-m$, then $\operatorname{co}_{n, k}\left(0^{m}\right)=\operatorname{co}_{n, k+i}(\rho)=0$ because there are not sufficiently many 0 s to make a pattern. Also, $\operatorname{co}_{n, k+i}(\rho)=0$ if $k<0$ since there are not enough rooks to make a pattern.

Consider each quantity in turn:

$$
\mathrm{co}_{n, k}\left(0^{m}\right)=\left(\begin{array}{l}
n \\
k
\end{array}\right)^{2} \cdot k !
$$

This comes from choosing $k$ columns and $k$ rows in which to place rooks and then permuting the rooks. Our range of $\mathrm{k}$ guarantees that there are at least $m$ empty columns, so all possible rook placements with $k$ rooks contain the pattern $0^{m}$. Also,

$$
\operatorname{co}_{n, k+i}(\rho) \geq\left(\begin{array}{c}
n \\
i
\end{array}\right) \cdot\left(\begin{array}{c}
n-i \\
k
\end{array}\right) \cdot\left(\begin{array}{c}
n-m \\
k
\end{array}\right) \cdot k !
$$

Here, $\operatorname{co}_{n, k}(\rho)$ is underestimated by counting only the rook placements that contain a copy of $\rho$ in their first $m$ positions. First, choose the rows of the $i$ rooks that will take part in the copy of $\rho$. Then of the remaining $n-i$ rows, choose $k$ to contain rooks in the remainder of the rook placement. Choose the columns of these rooks in one of $\left(\begin{array}{c}n-m \\ k\end{array}\right)$ ways, and then permute the $k$ rooks after the initial $\rho$ pattern in one of $k$ ! ways.

For fixed $m, i$, and $k,\left(\begin{array}{c}n \\ k\end{array}\right)^{2}$ is a polynomial in $n$ of degree $2 k$ with positive leading coefficient. Also, $\left(\begin{array}{c}n \\ i\end{array}\right) \cdot\left(\begin{array}{c}n-i \\ k\end{array}\right) \cdot\left(\begin{array}{c}n-m \\ k\end{array}\right)$ is a polynomial in $n$ of degree $2 k+i$ with positive leading coefficient. Since $i>0$, the latter quantity has a higher degree, and thus for sufficiently large $\mathrm{n}, \mathrm{co}_{n, k}\left(0^{m}\right) \leq \mathrm{co}_{n, k+i}(\rho)$.

This exhausts the computation for patterns of length at most three. The next section focuses on automated enumeration techniques for rook pattern avoidance.

\section{Enumeration schemes for rook patterns}

In general, our goal is to quickly compute $\mathrm{r}_{n}(Q)$ and $\mathrm{r}_{n, k}(Q)$ for various sets of rook patterns $Q$ and nonnegative integers $n$ and $k$. For sufficiently long 
patterns, these values become impossible to compute with a simple closed formula. Hence, more robust recursive techniques are needed.

An enumeration scheme is an encoding for a family of recurrences that enumerate the members of a family of sets. Enumeration schemes were first introduced and automated by Zeilberger [19] for pattern-avoiding permutations. Later, Vatter [18] improved the success rate of Zeilberger's enumeration schemes by introducing the notion of gap vectors, discussed below. Beyond permutations avoiding classical patterns, enumeration schemes have been extended to answer various other counting questions involving words avoiding permutations [14], words avoiding words [12], permutations avoiding vincular patterns [2], and permutations avoiding barred patterns [13]. Such schemes have two chief benefits. First, enumeration schemes can be deduced automatically by computer. Second, they allow computation of much more enumeration data than is feasible by brute force computation. More recently, Baxter [1] has extended enumeration schemes to enumerate pattern-avoiding permutations according to various statistics, and in certain cases Baxter uses schemes to derive a functional equation satisfied by the enumeration generating function he seeks.

This section provides an adaptation of previous enumeration scheme algorithms to work with pattern-avoiding rook monoid elements. Enumeration schemes are found via a divide-and-conquer method. First, the set to be enumerated is divided into several disjoint subsets using prefixes. Then two mechanisms help enumerate members of these subsets. Reversibly deletable elements provide recurrences that relate the size of subsets to simpler subsets. Gap pairs determine when a given subset is empty and may be discarded. Each of prefixes, reversibly deletable elements, and gap pairs may be determined completely algorithmically. The user need only input the set of patterns to be avoided and two positive integers used as search parameters; then either an enumeration scheme encoding is returned or the computer reports that no scheme was found with those particular search parameters. Each of the three main components of schemes will now be considered in greater detail.

\subsection{Prefixes}

Given a set of patterns $Q$, we wish to compute $\mathrm{r}_{n}(Q)$ and $\mathrm{r}_{n, k}(Q)$. First partition $\mathcal{R}_{n}(Q)$ into disjoint subsets. Given rook element $\pi \in \mathcal{R}_{n}$ and $i \leq n$, the $i$-prefix of $\pi$ is the pattern formed by the first $i$ entries of $\pi$. One simple way to partition $\mathcal{R}_{n}(Q)$ into disjoint subsets is by considering the $i$-prefix of each element. 
For example, $\mathcal{R}_{2}(00)=\{01,02,10,12,20,21\}$. When $i=1$, two of these elements (01 and 02) have 1-prefix 0, and the remaining elements (10, 12, 20 , and 21) have 1-prefix 1.

In general, write $\mathcal{R}_{n}^{p}(Q)$ for the set of $Q$-avoiding elements of $\mathcal{R}_{n}$ that have prefix $p$, and $\mathcal{R}_{n, k}^{p}(Q)$ for the subset of $\mathcal{R}_{n}^{p}(Q)$ with exactly $k$ nonzero elements. Similarly, $\mathrm{r}_{n}^{p}(Q)=\left|\mathcal{R}_{n}^{p}(Q)\right|$ and $\mathrm{r}_{n, k}^{p}(Q)=\left|\mathcal{R}_{n, k}^{p}(Q)\right|$.

Further, given a prefix $p$, the children of $p$, denoted $C(p)$, are the set of rook patterns of length $|p|+1$ whose $|p|$-prefix is $p$. For example, if $\epsilon$ is the empty word, then $C(\epsilon)=\{0,1\}, C(0)=\{00,01\}, C(1)=\{10,12,21\}$, and $C(210)=\{2100,2103,3102,3201\}$.

Note that $\mathcal{R}_{n}^{p}(Q)=\bigcup_{p^{*} \in C(p)} \mathcal{R}_{n}^{p^{*}}(Q)$, where the union is disjoint, and thus, after taking cardinalities, $\mathrm{r}_{n}^{p}(Q)=\sum_{p^{*} \in C(p)} \mathrm{r}_{n}^{p^{*}}(Q)$. In particular, for any set of patterns $Q$,

$\mathrm{r}_{n}(Q)=\mathrm{r}_{n}^{0}(Q)+\mathrm{r}_{n}^{1}(Q)=\left(\mathrm{r}_{n}^{00}(Q)+\mathrm{r}_{n}^{01}(Q)\right)+\left(\mathrm{r}_{n}^{10}(Q)+\mathrm{r}_{n}^{12}(Q)+\mathrm{r}_{n}^{21}(Q)\right)=\cdots$.

At times, this may not be enough information to determine recursive structure and it is necessary to explicitly give the first few digits of a rook placement rather than just their pattern. When this is the case, define a two-component notation as follows

$$
\mathcal{R}_{n}^{(p, w)}(Q)=\left\{\pi \in \mathcal{R}_{n}^{p}(Q) \mid \pi_{1} \cdots \pi_{|p|}=w\right\}
$$

For example, while $\mathcal{R}_{2}^{1}(00)=\{10,12,20,21\}, \mathcal{R}_{2}^{(1,2)}(00)=\{20,21\}$. This provides a way to further partition $\mathcal{R}_{n}(Q)$. In particular

$$
\mathcal{R}_{n}^{p}(Q)=\bigcup_{p^{*} \in C(p)} \bigcup_{w \sim p^{*}} \mathcal{R}_{n}^{\left(p^{*}, w\right)}(Q),
$$

where $w \sim p^{*}$ indicates that $w$ is order-isomorphic to $p^{*}$.

It is also straightforward to program the computer to partition $\mathcal{R}_{n}$ according to prefixes and initial subwords. Now consider the problem of recursively counting the members of these new smaller sets. Certainly some of these sets are easy to compute, for example if $p$ does not avoid $Q$, then $\mathrm{r}_{n}^{p}(Q)=0$, or if $p$ avoids $Q$ and $|p|=n$, then $\mathrm{r}_{n}^{p}(Q)=1$. But for more complicated cases, other techniques are required.

\subsection{Reversibly deletable elements}

How can $\mathrm{r}_{n}^{p}(Q)$ be computed recursively? Any rook element in $\mathcal{R}_{n}(Q)$ yields a rook element in $\mathcal{R}_{n-1}(Q)$ by choosing a rook and erasing its row and 
column. For example, erasing the row and column of the first rook in the rook element $2103 \in \mathcal{R}_{4}(00)$ yields $102 \in \mathcal{R}_{3}(00)$. Given prefix $p$ of length $\ell$ and integer $i$ where $i$ denotes the column position of a rook in prefix $p$, define the deletion map $d_{i}: \mathcal{R}_{n}^{p}(Q) \rightarrow \mathcal{R}_{n-1}^{p_{1} \cdots p_{i-1} p_{i+1} \cdots p_{\ell}}(Q)$ as the function that deletes the row and column of the rook in position $i$.

Clearly, erasing a rook cannot create a forbidden pattern. Sometimes, it turns out that $d_{i}$ is a bijection. To show this, it must be shown that reinserting a rook in column $i$ into a member of $\mathcal{R}_{n-1}^{p_{1} \cdots p_{i-1} p_{i+1} \cdots p_{\ell}}(Q)$ does not introduce a forbidden pattern from $Q$. Equivalently, by contrapositive, one can consider every element of $\mathcal{R}_{n}^{p}$ that contains a member of $Q$ and show that deleting the rook in column $i$, row $p_{i}$ still produces a rook placement that contains a pattern from $Q$. Again, it is possible to program the computer to generate the set of all scenarios where a rook placement contains a pattern from $Q$ involving column $i$ in a forbidden pattern, delete the rook, and check for containment in the new smaller rook placement, thus automatically determining if $d_{i}$ is a bijection. If $d_{i}$ is a bijection, then position $i$ is said to be reversibly deletable for prefix $p$ and pattern set $Q$.

For example, consider $Q=\{102\}$ and $p=12$. In this case, $d_{2}$ is a bijection from $\mathcal{R}_{n}^{12}(Q)$ to $\mathcal{R}_{n-1}^{1}$. In particular, if $\pi \in \mathcal{R}_{n}^{12}(Q)$, then $\pi_{1}<\pi_{2}$. If $\pi_{2}$ is involved in a forbidden 102 pattern, then $\pi_{2}$ plays the role of a 1 , so it is followed by $\pi_{i}=0$ and $\pi_{j}>\pi_{2}$ for some $2<i<j \leq n$. But removing $\pi_{2}$ gives $\pi_{1} \pi_{3} \cdots \pi_{n}$ where $\pi_{1}<\pi_{j}$, so $\pi_{1} \pi_{i} \pi_{j}$ is still a 102 pattern and position 2 is reversibly deletable. In this case, $\mathrm{r}_{n}^{\left(12, w_{1} w_{2}\right)}(102)=\mathrm{r}_{n-1}^{\left(1, w_{1}\right)}(102)$ for any $w \sim 12$.

With rook placements, one other scenario is possible. A smaller rook placement may be obtained by deleting an empty column. When this happens, there may be a choice of which corresponding row to delete. In fact, if an empty column is placed at the front of the rook placement $\pi \in \mathcal{R}_{n-1}$, then there are $n$ choices of where to insert an empty row.

Given prefix $p$ and pattern set $Q$, when $p_{i}=0$, position $i$ is reversibly deletable if there is no way for $p_{i}$ to be involved in a forbidden pattern from $Q$. For example, if $Q=\{102\}$ and $p=0$, position 1 is reversibly deletable. In the case of this rook pattern, however, this deletion does not give a bijection, but a many-to-one correspondence. In a 102-avoiding rook placement, there are $n$ ways to insert an empty row along with the empty first column to obtain a member of $\mathcal{R}_{n-1}(102)$. In this case, $\mathrm{r}_{n}^{(0,0)}(102)=n \mathrm{r}_{n-1}^{(\epsilon, \epsilon)}(102)$.

\subsection{Gap pairs}

While reversibly deletable elements may determine a recurrence for $\mathrm{r}_{n}^{p}(Q)$ in many cases, they are generally not sufficient for determining a complete 
system of recurrences. Thus, one more tool for computing these cardinalities is required.

Often, $\mathcal{R}_{n}^{(p, w)}(Q)=\emptyset$. Gap pairs encode which choices of $p, w$, and $Q$ produce such an empty set.

Our first step is to define gap vectors. Given a prefix $p$ of length $\ell$, and rook element $\pi$, let $\hat{\pi}_{1}<\hat{\pi}_{2}<\cdots<\hat{\pi}_{m}$ be the nonzero digits of $\pi$ involved in prefix $p$. In this case, $\pi$ is said to obey gap vector $v \in \mathbb{N}^{m+1}$ with respect to $Q$ if $\pi$ avoids $Q, \pi$ has prefix $p$, there are at least $v_{1}$ nonzero digits of $\pi$ after the prefix and less than $\hat{\pi}_{1}$, at least $v_{m+1}$ digits of $\pi$ after the prefix and greater than $\hat{\pi}_{m}$, and for $1<i \leq m$, there are at least $v_{i}$ digits of $\pi$ after the prefix and between $\hat{\pi}_{i}$ and $\hat{\pi}_{i+1}$. For example, $\pi=304201 \in \mathcal{R}_{n}^{(10,30)}(000)$ obeys the gap vector $\langle 2,1\rangle$ since 304201 has two digits (namely 1 and 2) smaller than $\hat{\pi}_{1}=3$ and one digit (4) that is larger than 3. Similarly, $\pi$ obeys the vector $\langle 1,1\rangle$, and any other vector in $\mathbb{N}^{2}$ that is componentwise smaller than $\langle 2,1\rangle$.

Given a prefix $p$ with $m$ nonzero digits and a set of forbidden patterns $Q$, a gap pair consists of a vector $v \in \mathbb{N}^{m+1}$ and a nonnegative integer $z$ such that there are no rook elements $\pi$ that avoid $Q$, have prefix $p$, obey $v$ and have at least $z$ empty columns appearing after the prefix.

For example, if $Q=\{102\}$ and $p=10$, then $[\langle 0,1\rangle, 0]$ is a gap pair because any rook element with one digit larger than the 1 contains a 102 pattern, i.e. $\mathcal{R}_{n}^{10}(102)$ contains no members obeying the gap vector $\langle 0,1\rangle$.

Similarly, if $Q=\{00\}$ and $p=01$, then $[\langle 0,0\rangle, 1]$ is a gap pair because any rook element with an additional empty column contains a 00 pattern, i.e. $\mathcal{R}_{n}^{01}(00)$ contains no members with a 0 after the initial prefix.

Gap pairs reduce the number of scenarios one must consider to determine if there are reversibly deletable elements. For example, the gap pair $[\langle 0,1\rangle, 0]$ for $Q=\{102\}$ and $p=10$ tells us that the role of 1 in the 10 prefix must be played by the highest rook. Therefore, this rook cannot be involved in a 102 pattern, and so position 1 is reversibly deletable.

Again, determining the set of minimal gap pairs for a given set of patterns and prefix becomes a matter of listing out all possible rook placements of minimum length that obey the gap vector and include an appropriate number of additional empty columns. If every single such rook placement contains a forbidden pattern, this yields a gap pair.

\subsection{Computing enumeration schemes}

All of the necessary pieces are now in place to give a formal definition of enumeration scheme. A rook enumeration scheme is a set $S$ of triples $[p, R, G]$ where 
1. $[\epsilon, \emptyset, \emptyset] \in S$.

2. For every $[p, R, G] \in S, R \subseteq\{1, \ldots,|p|\}$, and $G$ is a set of gap pairs with vectors of length $k+1$, where $k$ is the number of nonzero digits of $p$.

3. For any $[p, R, G] \in S$, either

(a) $R \neq \emptyset$ or

(b) $C(p)$ are all members of triples of $S$.

In other words, a rook enumeration scheme is a set of triples consisting of a prefix $p$, a set of reversible deletable elements for $p$, and a set of gap pairs for $p$ that includes the trivial prefix $\epsilon$, and such that every prefix in the scheme either has reversibly deletable elements or has all its children also appearing as prefixes in the scheme.

Given a set of forbidden patterns, one need only specify the maximum length prefix to consider and the maximum weight gap pair (here weight is the sum of the entries in the gap vector plus the integer $z$ ). The computer can then divide $\mathcal{R}_{n}(Q)$ into subsets according to prefixes, and then for each prefix determine the reversibly deletable elements and gap pairs. The two search parameters allow the user to control the amount of time spent searching for a scheme. If no scheme is found within the search parameters the computer reports the search has failed. If a pattern set has a scheme with maximum gap pair weight at most $w$ and depth at most $d$, then the pattern set is $(w, d)$-denumerable, or $(w, d)$-D for short.

For example, if $Q=\{102\}$, the scheme obtained has maximum gap weight 1 of depth 2 :

$S=\{[\epsilon, \emptyset, \emptyset],[0,\{1\}, \emptyset],[1, \emptyset, \emptyset],[10,\{1,2\},\{[\langle 0,1\rangle, 0]\}],[12,\{2\}, \emptyset],[21,\{1\}, \emptyset]\}$, so $\{102\}$ is $(1,2)-D$.

Notice that indeed, $[\epsilon, \emptyset, \emptyset] \in S$. For every triple with a nonempty set $G$, the vector has length one more than the number of nonzero entries of the corresponding $p$. Further, as $\epsilon$ and 1 have no reversibly deletable elements, $C(\epsilon)=\{0,1\}$ and $C(1)=\{10,12,21\}$ are prefixes in the scheme. For other prefixes there are indeed reversibly deletable elements.

The scheme above can be read as the following system of recurrences: $\mathrm{r}_{n}(102)=\mathrm{r}_{n}^{0}(102)+\mathrm{r}_{n}^{1}(102)$ (using the children of $\epsilon$ to partition). $\mathrm{r}_{n}^{0}(102)=n \mathrm{r}_{n-1}(102)$ (because the first position is reversibly deletable for the prefix $p=0)$. $\mathrm{r}_{n}^{1}(102)=\mathrm{r}_{n}^{10}(102)+\mathrm{r}_{n}^{12}(102)+\mathrm{r}_{n}^{21}(102)$ (using the children of 1 to partition). 
Table 2: Success rate of enumeration scheme algorithm

\begin{tabular}{|c|c|c|c|}
\hline Type & gap wt. w & depth d & $((\mathbf{w}, \mathbf{d})-D$ classes $) /($ Trivial classes $)$ \\
\hline$[1]$ & 1 & 1 & $2 / 2(100 \%)$ \\
\hline$[2]$ & 2 & 2 & $3 / 3(100 \%)$ \\
\hline$[3]$ & 3 & 3 & $7 / 7(100 \%)$ \\
\hline$[4]$ & 4 & 4 & $13 / 21(61.9 \%)$ \\
\hline$[2,2]$ & 2 & 2 & $5 / 5(100 \%)$ \\
\hline$[2,2,2]$ & 2 & 2 & $5 / 5(100 \%)$ \\
\hline$[2,3]$ & 3 & 3 & $16 / 16(100 \%)$ \\
\hline$[3,3]$ & 3 & 5 & $40 / 40(100 \%)$ \\
\hline
\end{tabular}

$\mathrm{r}_{n}^{10}(102)=\mathrm{r}_{n-1}^{0}(102)$ (because of the gap pair $[\langle 0,1\rangle, 0]$, the rook in the first column must be the highest rook and thus cannot be involved in a 102 pattern. Therefore the first column may be deleted.).

$\mathrm{r}_{n}^{12}(102)=\sum_{i=1}^{n-1} \sum_{j=i+1}^{n} \mathrm{r}_{n}^{(12, i j)}(102)=\sum_{i=1}^{n-1}(n-i) \mathrm{r}_{n-1}^{(1, i)}(102)$ (partitioning based on which particular rook placements can form a 12 prefix and using the fact that the second position is reversibly deletable for $p=12$ ).

$\mathrm{r}_{n}^{21}(102)=\sum_{i=1}^{n-1} \sum_{j=i+1}^{n} \mathrm{r}_{n}^{(21, j i)}(102)=\sum_{i=1}^{n-1}(n-i) \mathrm{r}_{n-1}^{(1, i)}(102)$ (partitioning based on which particular rook placements can form a 21 prefix and using the fact that the first position is reversibly deletable for $p=21$ ).

Thus

$$
\mathrm{r}_{n}(102)=n \mathrm{r}_{n-1}(102)+2 \sum_{i=1}^{n-1}(n-i) \mathrm{r}_{n-1}^{(1, i)}(102) .
$$

Similarly, the recurrence encoded by the scheme can be used to compute $\mathrm{r}_{n, k}(102)$ for any $0 \leq k \leq n$ using these same mechanisms which are tedious on paper, but easily done by computer.

\subsection{Success rate}

Table 2 contains the success rate of our enumeration scheme algorithm measured by trivial equivalence classes for which there exist a pattern set with a finite scheme. The output of the scheme program for each of these cases as well as the original enumeration scheme algorithm code can be found on the second author's website at http://faculty.valpo.edu/lpudwell/maple.html.

This $100 \%$ success rate with patterns of length at most three is not surprising since enumeration schemes have $100 \%$ effectiveness with permutation patterns of length at most three. Of the eight symmetry classes of length four patterns that failed, six are classes of permutation patterns for which there is no scheme and two are classes of rook patterns with zeros. The two 
non-permutation pattern classes with no scheme are 1320 and 1302. One can prove that there is no finite scheme for each of these patterns by exhibiting an infinite chain of prefixes with no reversibly deletable elements. For both 1320 and 1302 , such a chain is $1,12,231,3412,45231, \ldots,(2 i-2)(2 i-1)(2 i-$ 4) $(2 i-3) \cdots 1,(2 i-1)(2 i)(2 i-3)(2 i-2) \cdots 12, \ldots$

This success with patterns of small length is an effective way to quickly generate data for a variety of rook pattern classes quickly. Beyond confirming the results in Table 1, Table 3 gives a summary of the sequences obtained from avoiding sets of patterns of length two and three. Each result in this table has a corresponding enumeration scheme that can be used to verify the given formula; of course, alternate combinatorial arguments also exist. Far more of the sequences from pattern sets of type [3,3] and [4] are new to OEIS; data for these pattern sets are available at the second author's website.

Because of the fact that $\left|\mathcal{R}_{n}\right|>>n$ !, brute force computation is quite intensive. By brute force techniques, it takes several minutes to sort through the 130922 rook placements on a $7 \times 7$ board and consider whether each contains a given forbidden pattern. There are nearly 26 times as many elements as there are permutations of length 7 . Thus, computing data even for $n \leq 10$ is nontrivial. Enumeration schemes allow for this improved generation of data. Often, for small patterns, the initial data computed by an enumeration scheme leads to natural conjectures for a general formula that can then be proved by hand.

\section{Two-dimensional avoidance}

After exhaustively considering one-dimensional rook patterns of small length, it is time to briefly consider a two-dimensional type of rook pattern analogous to bivincular permutation patterns.

As before, a rook placement can be represented as a finite sequence $\pi_{1} \cdots \pi_{n}$ where $\pi_{i} \in\{0,1, \ldots, n\}$ and if $\pi_{i}=\pi_{j}>0$ then $i=j$. Before, the positions where $\pi_{i}=0$ were important; however only the relative order of nonzero digits was important, rather than their actual values. For example, in one-dimensional representation 10 and 20 are equivalent as patterns; both denote a single element followed by a 0 . However, in drawing these two patterns as rook placements, they appear distinct. 10 denotes a $2 \times 2$ board where the sole rook is in the bottom left cell, while 20 denotes a $2 \times 2$ board where the sole rook is in the upper left cell. Two-dimensional rook patterns capture this distinction. 
Table 3: Enumeration scheme sequences from avoiding a set of patterns

\begin{tabular}{|c|c|c|c|}
\hline Patterns $Q$ & $r_{n}(Q)$ & OEIS & Formula \\
\hline$\overline{12,21}$ & 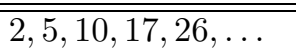 & $\overline{\mathrm{A} 002522}$ & $\overline{n^{2}+1}$ \\
\hline 00,12 & $2,5,10,17,26, \ldots$ & $\mathrm{A} 002522$ & $n^{2}+1$ \\
\hline 01,12 & $2,4,8,16,32, \ldots$ & A000079 & $2^{n}$ \\
\hline 01,10 & $2,3,7,25,121, \ldots$ & A038507 & $n !+1$ \\
\hline 00,01 & $2,4,12,48,240, \ldots$ & A052849 & $2 n !$ \\
\hline $00,12,21$ & $2,4,0,0,0, \ldots$ & A000004 & $0($ for $n \geq 3)$ \\
\hline $01,10,12$ & $2,2,2,2,2, \ldots$ & A007395 & 2 \\
\hline $01,12,21$ & $2,3,4,5,6, \ldots$ & $\mathrm{A} 000027$ & $n+1$ \\
\hline $00,01,12$ & $2,3,4,5,6, \ldots$ & $\mathrm{A} 000027$ & $n+1$ \\
\hline $00,01,10$ & $2,2,6,24,120, \ldots$ & $\mathrm{A} 000142$ & $n !($ for $n \geq 2)$ \\
\hline 01,120 & $2,5,13,39,151, \ldots$ & A127986 & $\overline{n !++2^{n}-1}$ \\
\hline 01,100 & $2,5,13,49,241, \ldots$ & A052898 & $2 n !+1($ for $n \geq 2)$ \\
\hline 01,123 & $2,5,15,51,188, \ldots$ & $\mathrm{A} 007317$ & $\sum_{i=0}^{n}\left(\begin{array}{c}n \\
i\end{array}\right)\left(\begin{array}{c}2 i \\
i\end{array}\right) /(i+1)$ \\
\hline 01,132 & & & equiv. to 01,123 \\
\hline 01,213 & & & equiv. to 01,123 \\
\hline 01,000 & $2,5,15,60,300, \ldots$ & new & $\frac{5}{2} n !$ \\
\hline 12,010 & $2,6,17,44,107, \ldots$ & new & $(n+1) 2^{n}-\frac{n 2^{n}}{2}-n$ \\
\hline 12,201 & & & equiv. to 12,010 \\
\hline 12,001 & & & equiv. to 12,010 \\
\hline 12,021 & & & equiv. to 12,010 \\
\hline 12,000 & $2,6,19,53,126, \ldots$ & $\begin{array}{c}\mathrm{A} 000537+ \\
\mathrm{A} 002522\end{array}$ & $\left(\frac{n(n-1)}{2}\right)^{2}+n^{2}+1$ \\
\hline 12,321 & & & equiv. to 12,000 \\
\hline 00,102 & $2,6,21,88,440, \ldots$ & new & $\begin{array}{c}n !+ \\
\sum_{i=1}^{n}(n(i-1) !(n-i) !)\end{array}$ \\
\hline 00,012 & $2,6,21,88,445, \ldots$ & A033540 & $a(n+1)=n(a(n)+1)$ \\
\hline 00,123 & $2,6,23,94,392, \ldots$ & $\begin{array}{c}\text { A037965+ } \\
\text { A000142 }\end{array}$ & $n !+n\left(\begin{array}{c}2 n-2 \\
n-1\end{array}\right)$ \\
\hline 00,132 & & & equiv. to 00,123 \\
\hline
\end{tabular}

Definition 2. Consider rook pattern $\rho \in \mathcal{R}_{m}$ as a placement of rooks on a $m \times m$ board. Rook placement $\pi \in \mathcal{R}_{n}$ is said to contain $\rho$ as a twodimensional pattern if there exist $m$ rows and $m$ columns of $\pi$ whose intersection forms $\rho$. Otherwise, $\pi$ avoids $\rho$.

Let $\mathrm{r}_{n, k}^{*}(Q)$ be the number of rook placements on an $n \times n$ board with exactly $k$ rooks that avoid all patterns in $Q$ in the two-dimensional sense, and let $\mathrm{r}_{n}^{*}(Q)=\sum_{k=0}^{n} r_{n, k}^{*}(Q)$. It is evident that $r_{n, k}^{*}(Q)=r_{n, k}(Q)$ if $Q$ is the all zeros pattern or if $Q$ consists only of permutations. However, patterns 
with some zero and some nonzero elements present a new challenge.

Brute force enumeration is certainly possible. One initial result: The number of rook elements avoiding 10 in the two-dimensional sense gives OEIS sequence A093345, which is also the number of $\{21, \overline{1} 2\}$-avoiding signed permutations in the hyperoctahedral group $B_{n}$ (see Section 2.3), as studied by Mansour and West in [10]. Avoiding $\{21, \overline{1} 2\}$ means that all unbarred numbers are in increasing order, and there is no smaller barred number followed by an unbarred larger number.

These sequences are the same by the following bijection from 10-avoiding rook monoid elements to $\{21, \overline{1} 2\}$-avoiding signed permutations.

Draw the rook monoid element as a rook placement on an $n \times n$ board. For each of the rooks, write the corresponding height with a bar over it. Fill in the empty columns with the remaining integers from $\{1, \ldots, n\}$ in increasing order. For example, $00 \mapsto 12,12 \mapsto \overline{12}, 21 \mapsto \overline{21}, 20 \mapsto \overline{2} 1$, $02 \mapsto 1 \overline{2}$, and $01 \mapsto 2 \overline{1}$.

All unbarred elements are in increasing order by construction. Further, avoiding 10 means no barred digit is followed by a larger unbarred digit, so this map sets each 10 -avoiding rook monoid element to a $\{21, \overline{1} 2\}$-avoiding signed permutation. The process is easily reversible: given a signed permutation, replace all unbarred numbers with zeros, and simply erase the bars from the barred elements.

Enumeration for two-dimensional patterns of length greater than two remains open and thus far has proved quite challenging.

\section{Acknowledgements}

The authors thank two anonymous referees for a number of valuable suggestions that were incorporated into the final draft of this paper.

\section{References}

[1] A. Baxter (2010). Refining enumeration schemes to count according to the inversion number, Pure Math. Appl. (PU.M.A.) 21(2) 137-160. MR2810530

[2] A. Baxter and L. Pudwell (2010). Enumeration schemes for vincular patterns, Discrete Math. 312 1699-1712. MR2901166

[3] A. Bjorner and F. Brenti (2005). Combinatorics of Coxeter Groups, Springer, New York. MR2133266 
[4] M. B. Can and L. E. Renner (2012). The Bruhat-Chevalley ordering on the rook monoid, Turkish J. Math. 36 499-519. MR2993581

[5] A. Claesson, V. Jelínek, E. Jelínková, and S. Kitaev (2011). Pattern avoidance in partial permutations, Electon. J. Combin. 18(1) P25 (41pp.) MR2770130

[6] E. Egge (2010). Enumerating rc-invariant permutations with no long decreasing subsequences, Ann. Comb. 14 85-101. MR2601802

[7] D. C. Fielder (2004). A generator of rook polynomials, Mathematica J. 9 371-385.

[8] T. Halverson and A. Ram (2004). q-rook monoid algebras, Hecke algebras and Schur-Weyl duality, J. Math. Sci. (N. Y.) 121(3) 2419-2439. MR1879072

[9] C. L. Liu (1968). Introduction to Combinatorial Mathematics, McGrawHill, New York, 111-118. MR0234840

[10] T. Mansour and J. West (2002). Avoiding 2-letter signed patterns, Sém. Lothar. Combin. 49 Article B49a (11pp.) MR1944848

[11] The On-Line Encyclopedia of Integer Sequences, published electronically at http://oeis.org, 2014.

[12] L. Pudwell (2008). Enumeration schemes for words avoiding patterns with repeated letters, Integers 8 A40. MR2472058

[13] L. Pudwell (2010). Enumeration schemes for permutations avoiding barred patterns, Electon. J. Combin. 17 R29. MR2595489

[14] L. Pudwell (2010). Enumeration schemes for words avoiding permutations. In Permutation Patterns, S. Linton, N. Ruskuc, and V. Vatter, Eds., Vol. 376 of London Mathematical Society Lecture Note Series, Cambridge University Press, 193-211. MR2732830

[15] J. Riordan (1958). An Introduction to Combinatory Analysis, Wiley, New York, 164-170. MR0096594

[16] J. R. Stembridge (1997). Some combinatorial aspects of reduced words in finite Coxeter groups, Trans. Amer. Math. Soc. 349(4) 1285-1332. MR1389789

[17] B. Tenner (2007). Pattern avoidance and the Bruhat order, J. Combin. Theory Ser. A 114 888-905. MR2333139

[18] V. Vatter (2008). Enumeration schemes for restricted permutations, Combin. Probab. Comput. 17 137-159. MR2376427 
[19] D. Zeilberger (1998). Enumeration schemes, and more importantly, their automatic generation, Ann. Comb. 2 185-195. MR1682929

DANIEL DALY

Department of Mathematics

Southeast Missouri State University

Cape Girardeau, MO, 63701

USA

E-mail address: ddaly@semo.edu

LARA PUdWEll

Department of Mathematics and Statistics

VALPARAISO UNIVERSITY

VALPARAISO, IN 46383

USA

E-mail address: Lara.Pudwell@valpo.edu

RECEIVED 6 JANUARY 2014 